\title{
EL ENVEJECIMIENTO DEL EMPRESARIADO AGRARIO EN EXTREMADURA. UNA APROXIMACIÓN MEDIANTE EL ANÁLISIS DE COMPONENTES PRINCIPALES
}

\author{
POR \\ FELIPE LECO BERROCAL
}

\section{Introducción}

El envejecimiento es uno de los problemas más graves con el que se enfrenta la Unión Europea y al que no escapa nuestro país, que ha seguido una tendencia evolutiva similar (Clarke, 1987).

Este proceso de envejecimiento es un fenómeno que tiende a generalizarse a todos los espacios geográficos, como ha puesto de manifiesto V. Rodríguez (1989) y otros tantos autores. A escala mundial, la población menor de quince años ha pasado del 33,5\% en 1985 al $32 \%$ en 1995, mientras que la población mayor de sesenta y cinco años era del $6 \%$ en uno y otro año (Aguilera, 1996).

En 1995 la población joven (menor de 15 años) en Europa era del $20 \%$, mientras que la población vieja (mayor de 65 años) ascendía al 13\%; en España esas cifras eran del $17 \%$ y del $15 \%$ respectivamente para el mismo año.

Pero es especialmente grave en aquellas áreas rurales, que han tenido que soportar una intensa emigración en las últimas décadas. De hecho, en estos momentos, se constituye como uno de los problemas esenciales al que se enfrenta el campo extremeño, tanto a nivel

Felipe Leco Berrocal. Departamento de Geografía y Ordenación del Territorio. Universidad de Extremadura. Cáceres

Estudios Geográficos

Tomo LVIII, n. ${ }^{\circ} 229$, octubre-diciembre 1997

$$
-625-
$$


estructural como por las negativas repercusiones socioeconómicas que ello conlleva.

Teniendo en cuenta los datos de los dos últimos Censos Agrarios, parece ser que en la década de los ochenta apenas se ha modificado el total de empresarios agrarios.

\section{Cuadro I}

EVOLUCIÓN DEL NÚMERO DE EMPRESARIOS AGRARIOS

\begin{tabular}{c|c}
\hline Años Censales & Total de empresarios \\
\hline 1982 & 114.892 \\
1989 & 114.615 \\
\hline
\end{tabular}

Fuente: Censos Agrarios, INE, Madrid.

En cambio, la composición estructural por edades sí se ha visto modificada; de este modo, en 1982 había un 52,1\% de empresarios menores de 54 años por un 46,1\% en 1989, mientras que los empresarios mayores de 65 años suponían un $21,7 \%$ en 1982 por un $24,3 \%$ en 1989 .

La distribución porcentual por edades y sexo de los empresarios agrarios en 1989 para el conjunto extremeño era la siguiente:

CUadro II

COMPOSICIÓN POR EDAD Y SEXO DEL EMPRESARIADO AGRARIO

EN EXTREMADURA, 1989

\begin{tabular}{c|c|c}
\hline Grupos de edad & Varones (\%) & Mujeres (\%) \\
\hline$<25$ & 0,85 & 0,33 \\
$25-29$ & 2,02 & 0,51 \\
$30-34$ & 3,50 & 0,80 \\
$35-39$ & 5,27 & 1,11 \\
$40-44$ & 7,15 & 1,50 \\
$45-49$ & 9,14 & 1,63 \\
$50-54$ & 10,50 & 1,81 \\
$55-59$ & 13,32 & 2,18 \\
$60-64$ & 11,80 & 2,25 \\
$>65$ & 18,23 & 6,12 \\
\hline
\end{tabular}

Fuente: Censo Agrario, INE, Madrid, 1989. 
A tenor de los datos anteriores, es evidente el progresivo envejecimiento de los empresarios agrarios en Extremadura (figura I). Al

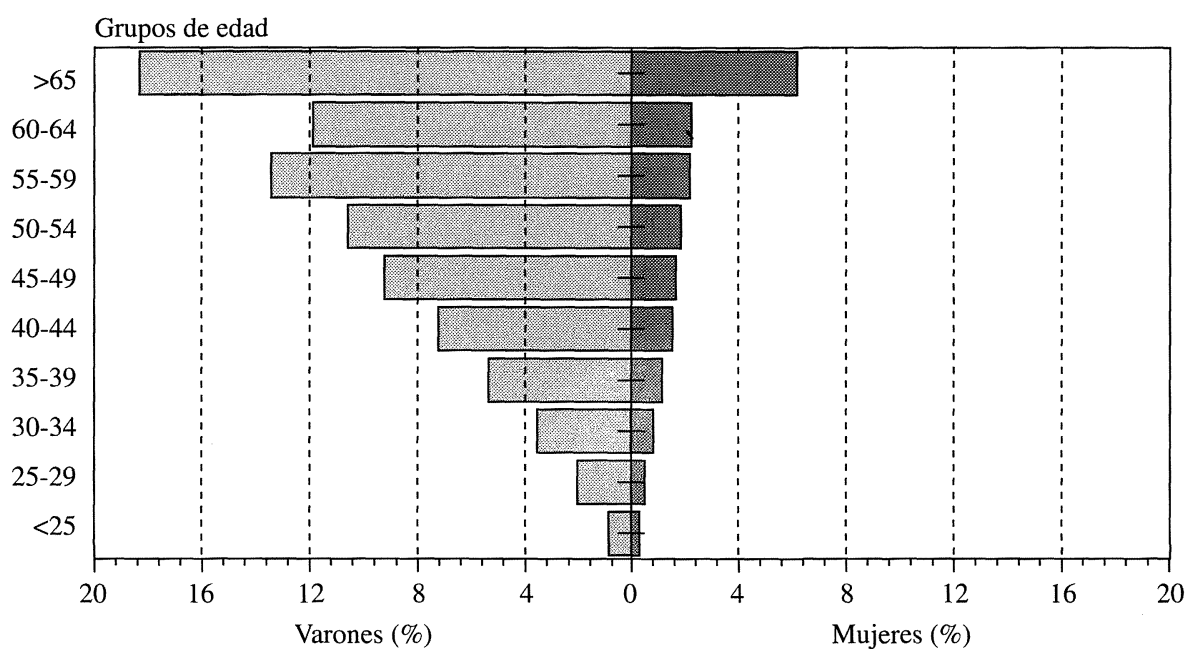

Fuente: Censo Agrario, 1989. INE, Madrid.

Figura 1.-Composición por edad y sexo del empresario agrario en Extremadura, 1989.

mismo tiempo, también es de destacar el peso de la mujer en el mundo rural, ya que más de un $18 \%$ de éstos son mujeres. Esta situación ha venido acompañada, además, por un paulatino abandono de las tierras labradas y un descenso del total de explotaciones, según se puede constatar en el cuadro siguiente.

Cuadro III

TIERRAS LABRADAS Y TOTAL DE EXPLOTACIONES EN EXTREMADURA

\begin{tabular}{|c|c|c|c|c|}
\hline \multirow{2}{*}{ Años Censales } & \multicolumn{2}{|c|}{ Explotaciones } & \multicolumn{2}{|c|}{ Tierras Labradas (ha) } \\
\hline & N. ${ }^{\circ}$ total & N. ${ }^{\circ}$ Índice & N. ${ }^{\circ}$ total & N. ${ }^{\circ}$ índice \\
\hline 1962 & 156.000 & 100,0 & 1.860 .464 & 100,0 \\
\hline 1982 & 103.425 & 66,3 & 1.277 .584 & 68,6 \\
\hline 1989 & 101.046 & 64,7 & 1.054.137 & 56,6 \\
\hline
\end{tabular}

Fuente: Censos Agrarios, INE, Madrid. 
De acuerdo con los datos precedentes, en las dos décadas del sesenta y del setenta, el total de explotaciones y las tierras labradas se han reducido en una tercera parte, coincidiendo con los momentos de mayor emigración. Si bien ambas variables parece que han evolucionado de forma paralela, no así en la última década, en la que las explotaciones han descendido moderamente, siguiendo las pautas de la emigración, mientras que las tierras labradas sufren un acusado descenso.

Este último aspecto se debe, sin duda, a numerosos factores: los efectos de la crisis y de las recesiones económicas, que han provocado el encarecimiento de los costes de producción; los negativos efectos de las sucesivas sequías; la falta de mano de obra y su encarecimiento, a pesar del elevado paro; la política de precios y la irregularidad del mercado; la PAC; el progresivo envejecimiento, etc., que han supuesto una mayor extensividad de los aprovechamientos agrarios.

Sirvan estos apuntes como introducción a la situación actual del campo extremeño y su evolución reciente, por cuanto que no es nuestro objetivo incidir sobre el tema del envejecimiento, ampliamente tratado ya (II Jornadas sobre la Población Española o en los sucesivos trabajos de Abellán, A., Rodríguez, V. y otros), ni siquiera sobre el análisis del evidente envejecimiento del empresario agrario extremeño, aspecto también tratado en diversas publicaciones del Departamento de Geografía y Ordenación del Territorio de la Universidad de Extremadura.

Nuestro objetivo fundamental es el de investigar si este proceso de envejecimiento se estaba produciendo de manera uniforme en todos los ámbitos rurales de la región.

Los contrastes espaciales del envejecimiento del empresariado agrario en Extremadura

Uno de los aspectos más importantes en el análisis del envejecimiento es su distribución y variación espacial (Warnes, 1987), ya que ello implica de hecho graves consecuencias sobre la economía y la estructura social de un área determinada (Paillat, 1987), así como profundas secuelas en la estructura por edades de la población (Bachiller, 1989). 
Al mismo tiempo, y como suele ocurrir con todas las manifestaciones de la dinámica geográfica, la homogeneidad no existe nunca (Aguilera, 1989). Por ello consideramos oportuno realizar una distribución espacial del empresariado agrario en Extremadura. Para llevar a cabo este análisis se generó una base de datos con los 381 términos municipales de Extremadura y distintas variables del Censo Agrario de 1989, entre las que se seleccionaron las correspondientes a la edad del empresariado por una parte, y las más características de cada uno de los diversos paisajes agrarios de la región (Leco,1995), por la otra: $\mathrm{X}_{01}$. Superficie de regadío, $\mathrm{X}_{02}$, Superficie de pastos y herbáceos, $\mathrm{X}_{03}$. Superficie de viñedo, $\mathrm{X}_{04}$. Superficie de frutales, $\mathrm{X}_{05}$. Superficie de olivar, $\mathrm{X}_{06}$. Superficie forestal, $\mathrm{X}_{07}$. Empresarios agrarios menores de 34 años, $\mathrm{X}_{08}$. Empresarios agrarios entre 35 y 54 años, $\mathrm{X}_{09}$. Empresarios agrarios mayores de 65 años, $\mathrm{X}_{10}$. Dedicación exclusiva.

Se trataba de clasificar básicamente los distintos municipios en función de su dedicación y de sus orientaciones principales y, en segundo lugar, las interrelaciones entre estas variables y la edad del empresariado.

Con esta finalidad se aplicó un análisis factorial en Componentes Principales (software Statview de Mac). Era necesaria una técnica multivariante que sintetizase la información en un número de variables mínimo e imprescindible (Santos, 1991), técnica que ya se había utilizado previamente -aunque con fines distintos- para la tipificación territorial del envejecimiento en Extremadura con buenos resultados (Gurría y otros, 1989).

La matriz de correlaciones, que se expone a continuación (Cuadro IV), permite una primera aproximación al objeto de estudio:

Por centrarnos en los objetivos planteados, únicamente reseñaremos que las áreas de regadío $\left(\mathrm{X}_{01}\right)$ tienen un empresariado agrario $\left(\mathrm{X}_{07} \mathrm{y} \mathrm{X}_{08}\right)$ más joven $(\mathrm{R}=0,279)$, por debajo de los 54 anos. Este mismo aspecto puede constatarse por la relación inversa de estas áreas de regadío con los empresarios de mayor edad $\left(\mathrm{X}_{09}\right)$, con un índice de $\mathrm{R}=-0,327$.

Por el contrario, las superficies de pastos, que en Extremadura ocupan más de la mitad de la SAU, con un aprovechamiento ganadero en régimen extensivo, reflejan unas correlaciones inversas a las del regadío. Tienen, por lo tanto, un empresariado considerablemente

$$
-629-
$$


CuAdro IV

MATRIZ DE CORRELACIÓN

\begin{tabular}{l|r|r|r|r|r|r|r|r|r|l}
\hline & \multicolumn{1}{c|}{$\mathbf{X}_{\mathbf{0 1}}$} & \multicolumn{1}{c|}{$\mathbf{X}_{\mathbf{0 2}}$} & $\mathbf{X}_{\mathbf{0 3}}$ & $\mathbf{X}_{\mathbf{0 4}}$ & $\mathbf{X}_{\mathbf{0 5}}$ & $\mathbf{X}_{\mathbf{0 6}}$ & $\mathbf{X}_{\mathbf{0 7}}$ & $\mathbf{X}_{\mathbf{0 8}}$ & $\mathbf{X}_{\mathbf{0 9}}$ & $\mathbf{X}_{\mathbf{1 0}}$ \\
\hline $\mathrm{X}_{01}$ & \multicolumn{1}{c|}{1} & & & & & & & & & \\
$\mathrm{X}_{02}$ & $-0,231$ & 1 & & & & & & & & \\
$\mathrm{X}_{03}$ & $-0,108$ & $-0,315$ & 1 & & & & & & & \\
$\mathrm{X}_{04}$ & 0,036 & $-0,055$ & $-0,063$ & 1 & & & & & & \\
$\mathrm{X}_{05}$ & $-0,271$ & $-0,075$ & 0,018 & 0,003 & 1 & & & & & \\
$\mathrm{X}_{06}$ & $-0,097$ & 0,064 & $-0,251$ & 0,049 & 0,055 & 1 & & & & \\
$\mathrm{X}_{07}$ & 0,279 & $-0,273$ & $-0,013$ & 0,133 & 0,035 & 0,014 & 1 & & & \\
$\mathrm{X}_{08}$ & 0,279 & $-0,344$ & 0,128 & 0,138 & $-0,014$ & $-0,034$ & 0,427 & 1 & & \\
$\mathrm{X}_{09}$ & $-0,327$ & 0,335 & $-0,129$ & $-0,107$ & 0,049 & 0,044 & $-0,603$ & $-0,785$ & 1 & \\
$\mathrm{X}_{10}$ & 0,108 & 0,246 & $-0,113$ & $-0,031$ & $-0,200$ & $-0,022$ & $-0,176$ & $-0,264$ & $-0,332$ & 1 \\
\hline
\end{tabular}

más envejecido, por cuanto que el índice de correlación con los mayores de 54 años es de $R=0,246$, mientras que este índice es inverso con los otros dos grupos de edades más jóvenes $(R=-0,273$ y $R$ $=-0,344$ respectivamente). Este empresariado «ganadero», más envejecido, muestra una mayor dedicación exclusiva a sus explotaciones.

Tras este primer acercamiento, se procedió a la realización del análisis factorial en Componentes Principales, mediante el método Ortogonal-Varimax y un procedimiento de «extracción de factor» que explicara el $100 \%$ de la varianza.

El resultado fueron tres factores, cuya explicación de la varianza fue la siguiente:

Cuadro V

EXPLICACIÓN DE LA VARIANZA

\begin{tabular}{c|c|c}
\hline Factores & Varianza Explicada (\%) & Varianza Acumulada (\%) \\
\hline I & 49,8 & 49,8 \\
II & 25,8 & 75,6 \\
III & 24,4 & 100,0 \\
\hline
\end{tabular}


Los tres factores, en los que se sintetiza y explica el 100\% de la realidad geográfica, contribuyen de manera importante a la definición de la estructura y del sistema agrario regional en función de las edades del empresariado, pero es el factor I el que adquiere una mayor importancia, al explicar prácticamente el $50 \%$ de esa realidad.

Las subestructuras que conforman cada uno de estos tres factores se definen en el Cuadro VI, que se expone a continuación. Entendemos por subestructura un conjunto de variables interrelacionadas entre sí por lazos de causalidad.

CuADRO VI

MATRIZ FACTORIAL

\begin{tabular}{l|c|c|c}
\hline Variables & Factor I & Factor II & Factor III \\
\hline Regadio & 0,446 & 0,663 & 0,021 \\
Pastos & $-0,553$ & 0,134 & 0,378 \\
Viñedo & 0,138 & $-0,207$ & $-0,785$ \\
Frutales & 0,232 & $-0,032$ & 0,328 \\
Olivar & $1.621 \mathrm{E}-3$ & $-0,738$ & 0,075 \\
Superficie forestal & $-0,018$ & $-0,216$ & 0,679 \\
Menos 34 años & 0,729 & 0,047 & 0,174 \\
De 35 a 54 años & 0,832 & 0,013 & $-0,027$ \\
Más de 65 años & $-0,898$ & $-0,036$ & $8.109 \mathrm{E}-3$ \\
Dedic. Exclusiva & -0423 & 0,5901 & 0,053 \\
\hline
\end{tabular}

Los tres factores aparecen definidos por subestructuras bipolares, caracterizando así a espacios agrarios distintos y contrapuestos:

1. El factor I se conforma, en primer lugar, por una subestructura característica del regadío (todas aquellas variables de signo positivo), y, en segundo lugar, por una subestructura que define a las áreas con predominio de los pastos y de la ganadería extensiva (variables con signo negativo).

Las áreas de regadío $(0,446)$ se caracterizan por un empresariado de edades intermedias, entre los 34 y los 54 años $(0,832)$, pero también por un empresariado joven, los menores de 34 años $(0,729)$. 
Por contraposición a esta subestructura de regadío, aparece la que caracteriza a las áreas de pastos $(-0,553)$, en las que la variable más definitoria es el empresariado con más de 65 años $(-0,898)$, que mantiene una dedicación agraria exclusiva $(-0,423)$.

En síntesis, podría afirmarse que en el regadío se está produciendo el relevo generacional, incorporándose personas jóvenes a las explotaciones. Ello puede deberse, en primer lugar, al mayor atractivo para los jóvenes de una agricultura más productiva; $\mathrm{y}$, en segundo lugar, a una agricultura muy mecanizada, que no exige una dedicación exclusiva, permitiendo otros trabajos remunerados en las ciudades que se localizan en estas áreas, bien en la construcción o bien, de forma estacional, en las industrias agroalimentarias.

En cualquier caso, habría que diferenciar a aquellos municipios de regadío con dedicación a tiempo parcial a sus explotaciones (factor I) y, a aquellos otros, definidos también por el factor II con una fuerte dedicación exclusiva en sus explotaciones (0,590). Estos submodelos serán analizados pormenorizadamente más adelante ya que, en primer término, podría resultar contradictorio.

En cambio, en las áreas de pastos se extiende una ganadería muy extensiva, poco productiva y muy dependiente de los factores climáticos y de las irregularidades e inestabilidad de los precios y del mercado. Su empresariado se encuentra muy envejecido, probablemente por la falta de aliciente para los jóvenes, que no tienen interés en continuar con esta actividad.

2. El factor II, también bipolar, como se ha indicado anteriormente, viene a reflejar las áreas dedicadas al monocultivo del olivar $(-0,738)$, y aquellas áreas de regadío $(0,663)$ con predominio de la dedicación exclusiva a las explotaciones agrarias $(0,590)$.

3. Por último, el factor III, se define por las áreas de monocultivo del viñedo $(-0,785)$ y, por otra parte, por las zonas con una agricultura de montaña, en la que el subsector forestal es el de mayor peso $(0,679)$, complementado por los pastos $(0,378)$ y por un minifundio agrícola destinado en gran medida a los frutales $(0,328)$.

Es evidente que, explicadas en su totalidad en el factor I las distintas variables de la edad del empresariado, ya no aparecen en los otros dos factores. Sin embargo, nuestro primer objetivo, era la clasificacióndelos distintos municipios en función de su dedicación agraria, para analizar más adelante su grado de envejecimiento, dado que 
el Análisis Factorial tan sólo descubría las áreas de mayor envejecimiento (las ganaderas) y las que contaban con un empresariado más joven (el regadío).

\section{Hacia una tipología de las áreas rurales}

De acuerdo con este primer objetivo y con los resultados estructurales del análisis factorial, se clasificaron todos los municipios, en función de que participaran en mayor o menor medida de cada una de las subestructuras reseñadas. Es decir, la clasificación tipológica de todos y cada uno de los términos municipales se realizó en función de sus pesos factoriales en la estructura general, para ello se consideró el umbral de $\pm 0,500$ en cada uno de esos factores. A grandes rasgos, pueden diferenciarse las siguientes áreas rurales (figura 2):

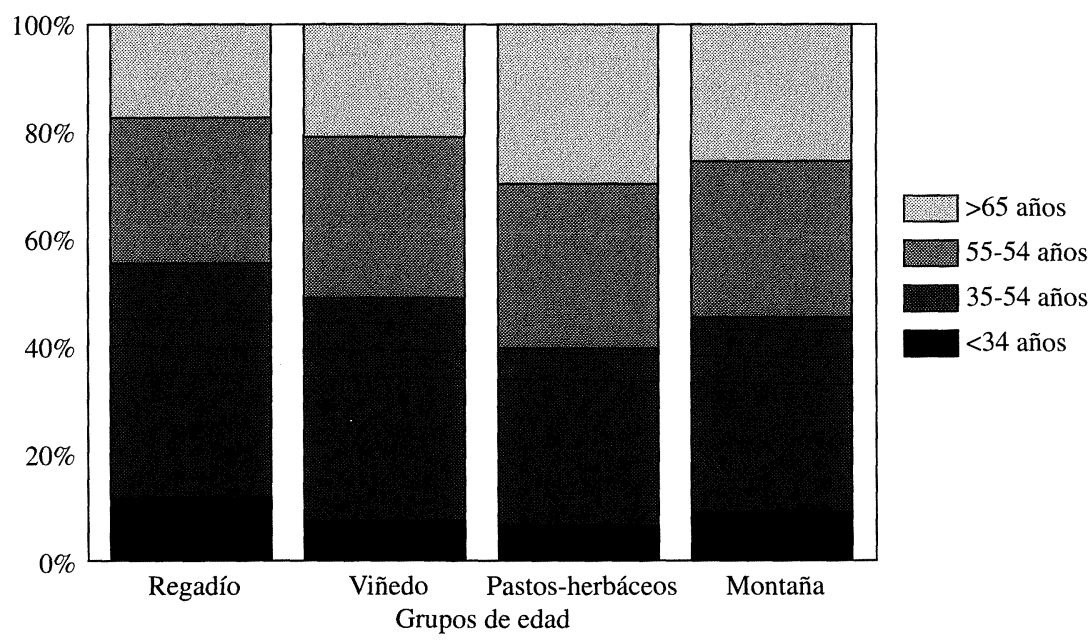

Fuente: Elaboración propia.

Figura 2.-Envejecimiento del empresariado agrario: Distribución por modelos agrarios.

El regadio, en el que se diferencia el regadío con poca dedicación exclusiva de los empresarios a sus explotaciones (factor I) y aquel otro regadío en el que se da una mayor dedicación agraria en exclusi- 
va (factor II). Se clasifican un total de 52 términos municipales que se localizan en las depresiones próximas al Sistema Central (Tiétar y Alagón-Arrago) y en las Vegas del Guadiana.

De estos 52 términos municipales, un total de 26 municipios se encuadran en el submodelo de regadío con una fuerte dedicación exclusiva en sus explotaciones. Estos municipios se localizan en torno a los regadíos de Coria (Cáceres) y Don Benito (Badajoz); áreas en las que existe una fuerte presencia de poblados de colonización y explican esa dedicación casi exclusiva en sus explotaciones.

En cambio, el resto de municipios queda encuadrado en un regadío a tiempo parcial más ligado a los regadíos de los valles de La Vera y El Jerte (Cáceres).

2. El conjunto de municipios caracterizados por los pastos y herbáceos ocupan una extensión bastante amplia, dado que quedan englobados todos aquellos con predominio de las superfcies adehesadas, de zonas desarboladas de pastos, y de zonas desarboladas con dedicación a los cereales de secano (factor I).

Conforman este subsistema agrario un total de 197 municipios, que básicamente se distribuyen por la penillanura cacereña (Llanos de Cáceres, Trujillo y Alcántara), Suroeste de Badajoz, La Serena y la Campiña de Llerena.

3. Áreas con monocultivo del viñedo (factor III), con un total de 42 términos municipales, que se distribuyen fundamentalmente por la Tierra de Barros (centro de la provincia de Badajoz) y algunos otros términos dispersos en la provincia de Cáceres (Cañamero, Montánchez, etc.).

4. Las áreas de montaña, que aparecen definidas en los tres factores, por cuanto que los distintos municipios no se clasifican en función de factores topográficos, sino en función de su dedicación. En este sentido, los municipios de montaña aparecen tipificados según su dedicación principal a los pastos y la ganadería extensiva (factor I), según su dedicación al monocultivo del olivar (factor II) o según una economía mixta, básicamente forestal-pastos-frutales (factor III). Participan de estas características estructurales un total de 91 términos municipales, que se localizan en el Sistema Central (Gredos-Hurdes-Gata), Villuercas y, en menor medida, Sierra Morena.

Una vez establecidos los distintos subsistemas agrarios en Extremadura, aunque únicamente por sus dedicaciones principales, se pro- 
cedió al estudio de las características del empresariado agrario correspondiente a cada uno de ellos y su grado de dedicación a sus correspondientes explotaciones.

Se procedió, por lo tanto, a contrastar la edad del empresariado por grandes grupos con cada uno de los modelos agrarios previos, poniéndose de manifiesto las diferencias entre unos y otros, según se recoge en el cuadro siguiente:

Cuadro VII

MODELOS AGRARIOS Y EDAD DEL EMPRESARIADO (\%)

\begin{tabular}{c|c|c|c|c|c|c|c}
\cline { 2 - 7 } & \multicolumn{3}{c|}{ Regadío } & \multicolumn{2}{c}{} \\
\hline Edad & Reg.1 & Media & Reg.2 & Viñedo & Pastos-Herb. & Montaña & Media \\
\hline$<34$ & 10,4 & 11,4 & 12,8 & 7,3 & 5,8 & 7,9 & 8,1 \\
$35-54$ & 44,1 & 44,9 & 45,6 & 40,2 & 33,3 & 37,6 & 39,0 \\
$55-64$ & 27,7 & 28,1 & 28,6 & 31,6 & 31,6 & 29,2 & 30,1 \\
$>65$ & 17,6 & 15,5 & 13,0 & 20,8 & 29,3 & 25,3 & 22,7 \\
Ded. Exc. & 60,7 & 63,3 & 66,8 & 55,8 & 60,6 & 54,5 & 58,5 \\
\hline
\end{tabular}

A la vista del cuadro precedente, casi una cuarta parte del empresariado cuenta con más de 65 años, es decir, casi 26.000 sobre los 114.615 existentes en Extremadura. Pero quizás lo más grave es que más de la mitad está por encima de los 55 años.

Por el contrario, los menores de 34 años únicamente suponen un $8,1 \%$, lo que denota la lenta y reducida incorporación de los jóvenes a las empresas agrarias. En unos casos, se debe a la falta de aliciente para los jóvenes de unas explotaciones reducidas y poco productivas, muy sometidas a las irregularidades climáticas y a la inestabilidad de precios y que exigen un trabajo muy sacrificado, lo que les lleva incluso al abandono después de incorporarse a las mismas. En otras ocasiones, son las mentalidades seculares y la organización de la familia, muy nuclear, que impide la incorporación de los jóvenes a las explotaciones hasta el fallecimiento del padre.

Igualmente, puede comprobarse que el envejecimiento y la edad del empresariado no son uniformes en los distintos «subsistemas» agrarios, presentando notables diferencias.

$$
-635-
$$


El regadío, que cuenta con más de 22.000 empresarios agrarios ( $20 \%$ del total), es el que presenta un mayor porcentaje, aun siendo bajo, de empresarios jóvenes (11,4\%), y una menor proporción, aun siendo elevada, de empresarios con más de 65 años (15,5\%). Los mayores de 55 años suponen el $43,6 \%$, un diez por ciento inferior a la media regional (figura 3 ). Dentro de los submodelos de regadío, el grupo correspondiente a dedicación exclusiva (Regadío 2) presenta un $66,8 \%$ de empresarios que trabajan única y exclusivamente en su explotación, al mismo tiempo cuentan con el porcentaje menor de empresarios mayores de 65 años de todos los modelos (13\%); mientras que el Regadío 1 sólo alcanza el 60\% de empresarios en dedicación exclusiva.

En las áreas con monocultivo de viñedo existen algo más de 12.000 explotaciones agrarias (un 10,8\% del total). Estas áreas se caracterizan por un empresariado más envejecido, dado que los mayores de 65 años suponen el 20,8\% y los de más de 55 años el 52,4\%. Aun siendo áreas envejecidas, no son las que presentan los mayores porcentajes. Pero, por el contrario, sí son las de menor proporción de empresarios jóvenes, conjuntamente con las zonas de Pastos-Herbáceos. Estos empresarios jóvenes vienen a ser la mitad de los existentes en el regadío (figura 2 ).

Los espacios agrarios con predominio de los pastos-herbáceos (de secano), con casi 55.000 explotaciones (prácticamente el $48 \%$ del total regional), son los que aparecen con un mayor envejecimiento, por cuanto que casi una tercera parte de los empresarios tiene más de 65 años, y casi las dos terceras partes más de 55 años. Por otra parte, son los de menor porcentaje de empresarios jóvenes, con tan sólo un 5,8\% con menos de 34 años (figura 2).

La montaña, finalmente, cuenta con algo más de 23.000 explotaciones (en torno a un $21 \%$ del total). Es el ámbito más envejecido, después de las zonas de pastos-herbáceos, dado que los mayores de 65 años representan más del $25 \%$ y los mayores de 55 el $54 \%$, en ambos casos por encima de las medias regionales. Pero, simultáneamente, también presentan unos índices mayores de empresarios jóvenes (8\%). Y es que hay que tener en cuenta que, dentro de este conjunto, se da una considerable dispersión en cuanto a su dedicación y extensión de cada uno de los tres subsectores (forestal-pastos-cultivos), y especialmente en cuanto a la dedicación de sus tierras de cultivo (se- 


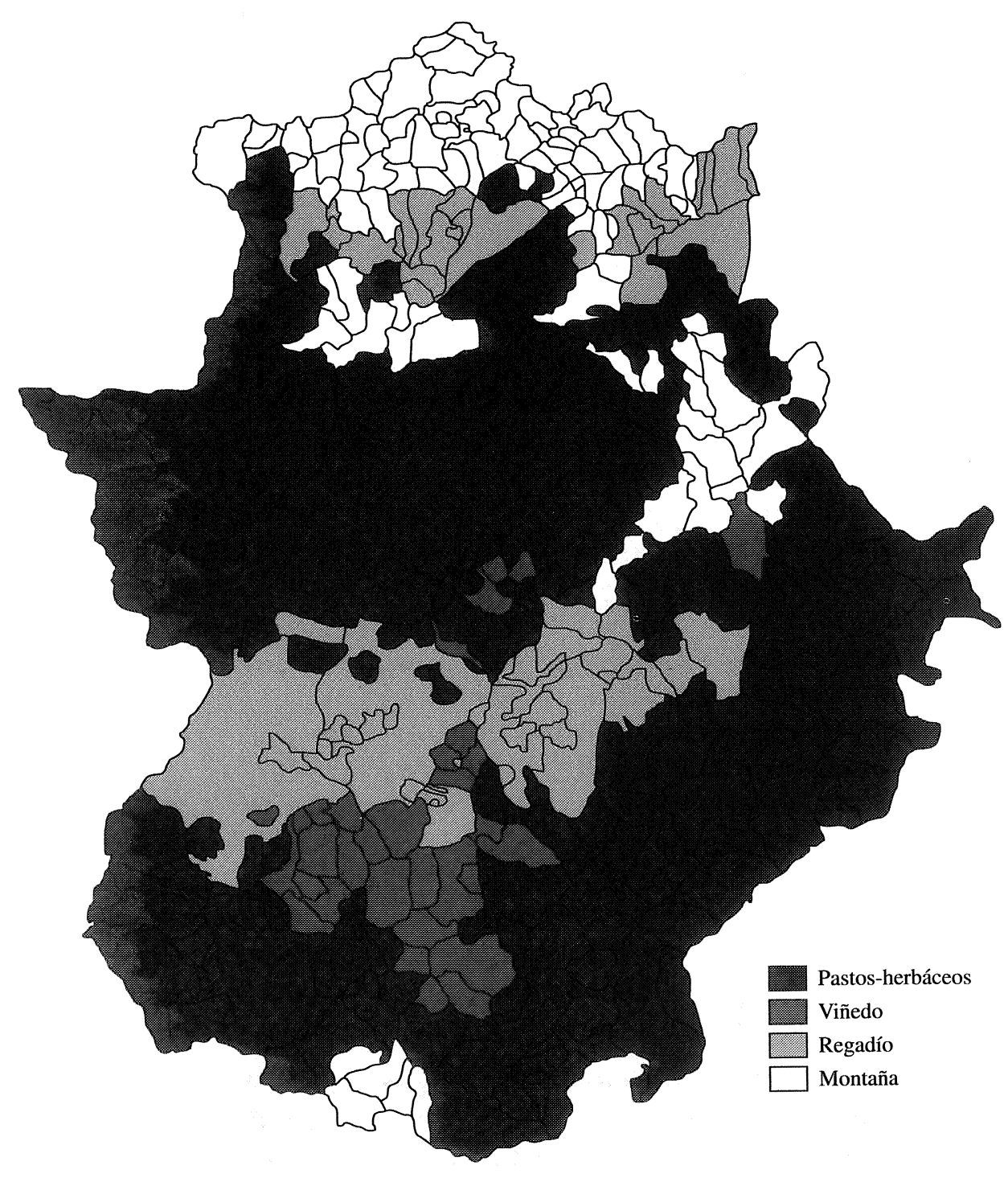

Figura 3.-Modelos agrarios en Extremadura.

$-637-$ 
cano-regadío, frutales-olivar-herbáceos) y su rentabilidad (agricultura de subsistencia-agricultura de mercado). Estas, al menos aparentes, contradicciones tienen fiel reflejo en la composición estructural de los empresarios por grupos de edades.

En cuanto a la dedicación agraria de este empresariado, son el regadío y los pastos los que ocupan en exclusiva a un mayor porcentaje. En el caso del regadío hemos convenido diferenciar el regadío según el factor I y según el factor II: el primero tiene una baja dedicación exclusiva y el segundo mucha. En el caso de los pastos y la ganadería extensiva, a pesar de las bajas exigencias en mano de obra, se debe a la avanzada edad de los empresarios y al mayor tamaño de las explotaciones.

Cuadro VIII

MODELOS AGRARIOS Y EDAD DEL EMPRESARIADO (TOTALES)

\begin{tabular}{c|r|r|r|r|r|r|r}
\cline { 2 - 7 } & \multicolumn{3}{c|}{ Regadio } & \multicolumn{1}{c}{} \\
\hline Edad & Reg.1 & Media & Reg.2 & Viñedo & Pastos-Herb. & Montaña & Media \\
\hline$<34$ & 1.264 & 2.488 & 1.224 & 971 & 3.301 & 2.330 & 9.090 \\
$35-54$ & 5.921 & 10.332 & 4.471 & 4.896 & 18.899 & 9.179 & 43.306 \\
$55-64$ & 3.821 & 6.536 & 2.715 & 3.804 & 16.840 & 6.444 & 33.624 \\
$>65$ & 2.569 & 3.903 & 1.334 & 2.706 & 15.685 & 5.509 & 27.803 \\
Ded. Exc. & 8.717 & 15.291 & 6.574 & 7.005 & 33.317 & 12.445 & 88.058 \\
\hline
\end{tabular}

En el cuadro precedente, podemos destacar el importante peso del empresariado agrario en el modelo de Pastos-Herbáceos que, al mismo tiempo, era uno de los más envejecidos, con menor posibilidad de reemplazo generacional y una mayor dedicación exclusiva $(60,6 \%)$.

El modelo de Regadío presenta características contrarias, a pesar de su fuerte dedicación exclusiva $(63,3 \%$, ya que tiene un grupo incipiente de empresarios agrarios jóvenes (menos de 34 años) lo que, en primera instancia, posibilita el reemplazo generacional.

En definitiva, esta situación de progresivo envejecimiento del empresariado agrario es bastante grave en Extremadura, ya que todavía 
más de un $20 \%$ de la población activa extremeña pertenece a la agricultura.

CuAdro IX

POBLACIÓN OCUPADA EN EL SECTOR AGRARIO, 1993

\begin{tabular}{c|c|c}
\hline Año & Extremadura (\%) & España (\%) \\
\hline 1960 & 68,8 & 40,1 \\
1973 & 48,8 & 25,5 \\
1981 & 35,3 & 21,1 \\
1986 & 30,2 & 17,8 \\
1993 & 19,3 & 12,2 \\
\hline
\end{tabular}

Fuente: Anuarios Estadisticos de la Junta de Extremadura, Mérida.

Como se puede observar en el cuadro precedente, todavía existe en Extremadura un alto porcentaje de población ocupada en el sector agrario, indicador que pone en evidencia la dependencia de dicho sector de la región; a pesar de ello, la evolución ha seguido las pautas del comportamiento nacional aunque, eso sí, con valores mucho más elevados (figura 4).

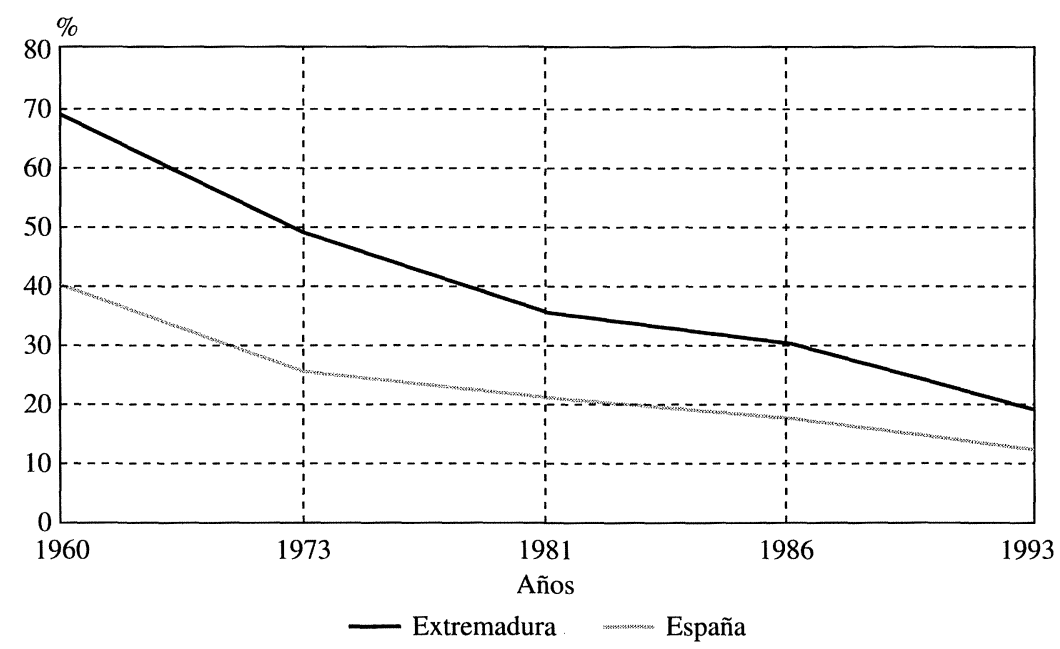

Fuente: Anuarios Estadísticos de la Junta de Extremadura.

FIGURA 4.-Población ocupada en el sector agrario: Extremadura vs. España.

$$
-639-
$$


Por otro lado, las actuales directrices de la PAC, que están propiciando el abandono del campo, no están favoreciendo la incorporación de los jóvenes al sector agrario puesto que no encuentran atractiva dicha opción.

Al mismo tiempo, estos aspectos se ven acompañados de una falta de dinamismo y espíritu emprendedor de un empresariado envejecido que no moderniza ni se sube al progreso técnico actual (Abellán, 1989), salvo en aquellas áreas más dinámicas (Regadío y Viñedo) que seguirán marcando cada vez diferencias más fuertes con el resto de modelos.

\section{Conclusiones}

Cabría significar, en primer lugar, las fatales consecuencias que provocará en un futuro próximo la ausencia de un reemplazo generacional de empresarios en la agricultura extremeña a nivel general, si bien hemos podido comprobar que existen modelos agrarios, más productivos y rentables (como el regadío y el viñedo), cuyo nivel de envejecimiento es menos grave.

Las políticas regionales, a tenor de los resultados obtenidos, deben ser inmediatas $\mathrm{y}$, ante todo, prácticas y promovedoras de iniciativas de incorporación de los jóvenes a la agricultura, a sus modos de producción actuales y siguiendo las pautas de las nuevas directrices de la PAC en materia de agricultura ecológica, turismo rural, extensividad de las producciones y manejo de razas ganaderas autóctonas, entre otras.

En este sentido, la Junta de Extremadura acaba de dar un primer paso para «rejuvenecer» el campo extremeño, mediante el cese anticipado de agricultores en Extremadura.

Los beneficiarios de estas nuevas medidas podrían ser todos los titulares de explotaciones con sesenta años de edad, que durante los últimos diez años hayan sido agricultores a título principal y que, al mismo tiempo, hayan cotizado a la Seguridad Social durante al menos quince años.

Las personas a las que se les cedan las explotaciones deberán tener menos de 50 años, deben estar dados de alta en el Régimen Especial Agrario y realizar la actividad agraria a título principal y 
deberán adquirir el compromiso de mantener esas explotaciones durante al menos cinco años.

Se estipula que cada uno de los agricultores que cedan sus explotaciones perciban unas ayudas de 16.000 ptas. por ha cedida, sin sobrepasar nunca las 500.000 ptas. por persona/año, además aquellos que se acojan a este decreto cobrarán anualmente unas 520.000 ptas. hasta cumplir la edad de jubilación definitiva.

Una excelente medida que, en la realidad, no parece ir acompañada de un paquete económico de compensación interesante para que los agricultores cedan sus explotaciones.

En todo caso, y a pesar de estas medidas, el problema del envejecimiento del empresariado agrario no es más que una prolongación del envejecimiento global de la población extremeña, pero también española, europea y mundial, como ya se reflejó en la introducción. Dado que el problema es evidente, aquellas medidas que inciten la incorporación de los jóvenes a la agricultura parece que, hoy día, pueden ser las más eficaces, sobre todo en el mundo rural extremeño dada la fuerte dependencia existente del sector agrario que, como ya se mencionó, cuenta con cerca del $20 \%$ de la población activa total ocupada en el sector agrario, cuando a nivel nacional esos valores se encuentran en torno a un $10 \%$.

\section{BIBLIOGRAFIA}

Abellan, A. (1989): El envejecimiento de la población. Tendencias e implicaciones. CSIC. Instituto de Economía y Geografla Aplicada. Departamento de Geografla Humana y Regional.

-, et al. (1989): «Proceso de envejecimiento de la población española, 1970-1981», en Análisis y desarrollo de la población española de 1970 a 1986. Madrid. Síntesis, pp. 357-364.

Aguilera Arilla, Ma a J., et al. (1989): «Los contrastes del envejecimiento demográfico entre el municipio de Madrid y su comunidad autónoma». II Jornadas sobre Población Española. Universidad de las Islas Baleares, pp. 67-79.

- (1996): «El envejecimiento de la población en Aragón». Estudios Geográficos, LVII, n. ${ }^{\circ} 225$. CSIC. Instituto de Economía y Geografía. Madrid, pp. 573-595.

Bachlller Martínez, J. M. a et al. (1989): «La provincia de Soria: Un caso extremo de envejecimiento demográfico». II Jornadas sobre Población Española. Universidad de las Islas Baleares, pp. 80-88.

Censos Agrarios de España, 1962-1972-1989. I.N.E. Madrid.

Clarke, J. (1987): «Le vieillissement en Europe: quelques avant-propos». Espace, Populations, Sociétés, 1. pp. 23-28. 
GURRÍA GASCÓN, J. L., et al. (1989): «El envejecimiento de la población extremeña. Aproximación metodológica». II Jornadas sobre Población Española. Universidad de las Islas Baleares, pp. 89-108.

-, et al. (1989): «Hacia la interpretación del envejecimiento en la población extremeña». II Jornadas sobre Población Española. Universidad de las Islas Baleares, pp. 89108.

Leco Berrocal, F. (1995): Los paisojes agrarios extremenos. Dpto. de Geografía y Ordenación del Territorio. Universidad de Extremadura, Cáceres.

PaIllat, P. (1987): «Régions à problèmes démographiques concernant la structure de la population visant en particulier le vieillissement». Actes du Seminaire sur le régions a problèmes démographiques en Europe. Strasbourg, pp. 305-395.

Rodrfguez, V., et al. (1989): Tipología del envejecimiento de la población española (19001986). CSIC. Instituto de Economía y Geografía Aplicada. Departamento de Geografía Humana y Regional.

Santos Preciado, J. M. (1991): «La técnica factorial y su aplicación en el campo geográfico». Espacio, Tiempo y Forma, Serie VI, Geografía, t. IV. Revista de la Facultad de Geografia e Historia. UNED, Madrid, pp. 1378.

Warnes, A. (1987): «La population agée du Royaume-Uni et sa distribution». Espace, Populations, Sociétés, 1, pp. 41-56.

RESUMEN: El fuerte envejecimiento actual del empresariado agrario en Extremadura supone, entre otras cosas, una importante pérdida de fuerza laboral en el mundo rural; aspecto que muestra claras diferencias dentro de los paisajes agrarios extremeños. Ante esta situación y para atenuar esta grave situación, se han instrumentado diversas medidas para incentivar el abandono de la producción de aquellos empresarios más envejecidos y posibilitar la incorporación de los jóvenes al sector agrario.

Palabras ClaVe: Empresariado agrario, envejecimiento, tipologías rurales, políticas regionales.

ABSTRACT: Actually, the strong ageing of agrarian business in Extremadura suppose an important lost in the laboral market in rural world, but this aspect is diferent for the diversus extremenian landscape. This situation has produced differents solutions to minimize this problem, some measures has been taken to incentive the abandonment of prodution for oldest business and to possibilite the young people's incoporation to agrarian sector.

KEY WORDS: Agrarian business, ageing, rural tipology, regional politic. 